\title{
Evaluation of immature granulocyte parameters in myeloid neoplasms assayed by Sysmex XN hematology analyzer
}

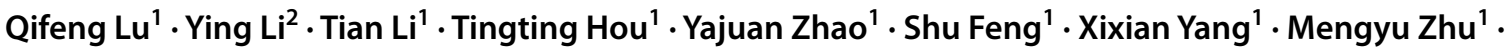 \\ Yajuan Shen ${ }^{1}$
}

Received: 5 October 2021 / Accepted: 25 January 2022 / Published online: 8 February 2022

(c) The Author(s) 2022

\begin{abstract}
Immature granulocytes (IGs) have significance for the diagnosis of myeloid neoplasms (MNs). The current study aims to use a hematology analyzer to evaluate the accuracy of IG parameters in MNs. Blood specimens from 388 patients with MN, 524 with non-hematological neoplasms (non-HNs), including 109 patients with inflammation and 68 undergoing G-CSF administration, and 500 healthy control subjects were analyzed. IG parameters was assayed by Sysmex XN-9000 (XN) and compared with manual assessments. A high level of agreement between IG\% derived from XN and manual measurements for MN patients $(r=0.828, p<0.0001)$ was revealed but only a moderate correlation for acute myeloid leukemia patients (AML; $r=0.597 ; p<0.0001$ ). Bland-Altman bias analysis was conducted, and the results showed that differences in IG\% from XN and manual analysis for MN patients were considered clinically insignificant. ROC analysis demonstrated a good performance of IG\# $($ AUC $=0.842)$ and IG\% $(\mathrm{AUC}=0.885)$ assessed by XN for MN patients with cut-off values of $0.200 \times 10^{9} / \mathrm{L}$ and $1.95 \%$, respectively. IG parameters from Sysmex XN analyzer are helpful for screening of MNs even though granulocyte morphological abnormalities may interfere with IG parameter accuracy.
\end{abstract}

Keywords Immature granulocytes $\cdot$ Myeloid neoplasms $\cdot$ Hematology analyzer $\cdot$ Morphological abnormalities

\section{Introduction}

Immature granulocytes (IGs) comprise promyelocytes, myelocytes, and metamyelocytes [1]. Such cells are seldom seen in the peripheral blood (PB) of healthy subjects, except in certain physiological states and conditions, such as pregnancy or the neonatal period [2-4]. By contrast, IGs are found in patients' $\mathrm{PB}$ in the following pathological conditions: acute infection, inflammation, tissue injury, intravascular hemolysis, acute hemorrhage, non-hematopoietic malignant tumor, hematological neoplasms and in those receiving granulocyte colony-stimulating factor (G-CSF) or granulocyte-macrophage colony-stimulating factor

Yajuan Shen

shenyajuanchina@126.com

1 Department of Clinical Laboratory, Shandong Provincial Hospital Affiliated to Shandong First Medical University, Jinan 250021, Shandong Province, China

2 Department of Hematology, Shandong Provincial Hospital Affiliated to Shandong First Medical University, Jinan 250021, Shandong Province, China
(GM-CSF) [5-13]. For patients undergoing G-CSF or GMCSF treatment, IGs act as sensitive indices to indicate granulocytic hematopoietic recovery [12, 13]. IGs are also seen in patients with myeloid neoplasms (MNs), such as acute myeloid leukemia (AML), myeloproliferative neoplasms (MPN), myelodysplastic syndrome (MDS), and myelodysplastic/myeloproliferative neoplasms (MDS/MPN), and their presence can be used for diagnosis. The conditions of chronic myeloid leukemia (CML) and primary myelofibrosis (PMF) are particularly associated with an increase in IG level [11].

Prior to the availability of the automated hematology analyzer, Sysmex XN series, other analyzers, including Sysmex $\mathrm{XS}, \mathrm{XT}$, and XE series, were used to sort cells for research purposes [6, 14]. The Sysmex XN analyzer uses one of the six white blood cell (WBC) differential parameters in the WBC differential (WDF) channel to sort IGs. Erythrocytes are lysed by lysercell WDF which permeates the leukocyte membrane to allow fluorescent staining of nucleic acids by fluorocell ${ }^{\mathrm{TM}} \mathrm{WDF}$. A semiconductor laser is then used to detect cells by flow cytometry. A combination of side scatter light (SSC), reflecting complexity of intracellular structures, 
side fluorescence light (SFL), reflecting fluorescence intensity, and forward scatter light (FSC), to measure cell size, is combined by an intelligent gating algorithm provided by 00-19E (Build 2) IPU version software to judge WBC type. The WDF scattergram shows IGs as dark blue dots above neutrophils. The Q-flag represents the healthy maximal limit of IGs and, above this threshold, "IG present" is indicated. Previous studies have reported general evaluation of Sysmex $\mathrm{XN}$ performance in measuring IG parameters [15-17], but application of this approach to MNs has been neglected.

The current study aimed to investigate Sysmex XN series performance in IG quantification in various MNs by comparison with manual measurements. Evaluation of the suitability of the Sysmex XN analyzer in reliable detection of IGs in PB and screening for MNs was assessed.

\section{Materials and methods}

\section{Blood samples}

A total of 1412 blood samples, including 388 from MN patients, 524 from patients with non-hematological neoplasms (non-HNs), and 500 from healthy control subjects, taken between November 2016 and December 2019 in Shandong Provincial Hospital Affiliated to Shandong First Medical University, Jinan, China, were included in the study.

MN patients were enrolled from the Department of Hematology and diagnosed according to 2016 WHO classification criteria [11, 18]. Specimens were collected at first diagnosis prior to chemotherapy or recurrence. Data from each individual was included in the study only once and MN patients without complete data were excluded. Characteristics of all MN patients are shown in Table 1. Non-HN patients were enrolled from departments other than hematology, including 284 from internal medicine, 177 from surgery, and 63 from ophalmology, othlaryngology, stomatology, gynecology, neurology, dermatology, or traditional Chinese medicine. Non-HN patients included 109 with inflammation from respiratory medicine, intensive care unit, and rheumatology and surgery department, and 68 undergoing recombinant human G-CSF administration from the oncology department. Healthy individuals were enrolled from the physical examination center with the exclusion of pregnant women.

Anticoagulant PB samples were taken with EDTA (Becton Dickinson, San Jose, USA) for routine blood analysis. The study was approved by the medical Ethics Committee of Shandong Provincial Hospital (No.2016-KY-051).

\section{Sample analysis}

The fully automated Sysmex XN series employs the automated hematology analyzer, XN-9000 (Sysmex, Kobe,
Table 1 Characteristics of MN patients

\begin{tabular}{ll}
\hline Diagnosis & Cases \\
\hline AML and related precursor neoplasms & 231 \\
AML with recurrent genetic abnormalities & 83 \\
AML with RUNX1-RUNX1T1 & 7 \\
AML with CBFB-MYH11 & 10 \\
Acute promyelocytic leukemia with PML-RARA & 42 \\
AML with mutated NPM1 & 10 \\
AML with biallelic mutation of CEBPA & 10 \\
AML with mutated RUNX1 & 4 \\
AML with myelodysplasia-related changes & 5 \\
Therapy-related AML & 3 \\
AML, NOS & 140 \\
AML without maturation & 6 \\
AML with maturation & 52 \\
Acute myelomonocytic leukemia & 41 \\
Acute monoblastic and monocytic leukemia & 41 \\
Myeloproliferative neoplasms & 77 \\
CML & 47 \\
Primary myelofibrosis & 30 \\
MDS & 63 \\
Myelodysplastic/myeloproliferative neoplasm & 17 \\
Chronic myelomonocytic leukemia & 10 \\
Myelodysplastic/myeloproliferative neoplasm, unclassifi- & 7 \\
able & \\
Total MN & 388 \\
\hline
\end{tabular}

Japan), equipped with 00-19E (Build 2) IPU version and the automated slide making/staining device, Sysmex SP-10 (Sysmex, Kobe, Japan). Annual calibration of the XN analyzer was performed, and precision, linearity, carryover, and stability were evaluated regularly. Measurement uncertainty, the dispersion of the measured value reasonably assigned by the characterization, was $0.03 \times 10^{9} / \mathrm{L}$ for IG\# from $\mathrm{XN}$, $0.2 \%$ for IG\% from $\mathrm{XN}$, and $0.8 \%$ for IG\% from manual methods. Two levels of blood XN check ${ }^{\mathrm{TM}}$ were conducted daily to monitor equipment performance and incorporated into the Sysmex program for real-time quality control. All $\mathrm{MN}$ and non-HN PB samples were initially analyzed by XN-9000; blood smears were made and stained using SP-10 and Wright Giemsa (Muto Pure Chemicals, Tokyo, Japan). Control samples were assayed by $\mathrm{XN}-9000$, only, and no blood smears made. All samples were analyzed within $4 \mathrm{~h}$ of collection. All threshold values of Q-flags ("IG present", "Left shift", "Blast", "Abn lympho", and "Atypical lympho") were set at 100. The WBC\#, RBC\#, HGB\#, PLT\#, IG\#, IG\%, instrument flags, and WDF scattergrams of all samples were recorded.

Two experienced technologists manually classified 200 cells per smear using an Olympus BX53 microscope according to the Clinical and Laboratory Standards Institute (CLSI) 
H20-A2 [19]. The mean IG\%, composed of promyelocytes, myelocytes, and metamyelocytes, calculated by the 2 technologists was considered to be the final result. In the event of significant differences between the results from the 2 technologists, a third technologist classified the slide.

\section{Statistical analysis}

Non-parametrical tests (Mann-Whitney test) were performed to compare IG parameters between 2 different groups and the Kruskal-Wallis test to compare 3 or more groups. Spearman rank correlation coefficient analysis was used to evaluate the relationship between IG\% values obtained from the Sysmex $\mathrm{XN}$ analyzer and from manual assessment. Values for $r$ coefficients $<0.30$ were considered to present negligible correlations; $0.30-0.50$ low correlations; $0.50-0.70$ moderate correlations; $0.70-0.90$ strong correlations; and $\geq 0.90$ a very high correlation [20]. Bland-Altman bias analysis was performed to compare IG\% differences from the two methods. Receiver operating characteristic (ROC) curve analysis was conducted to estimate sensitivity, specificity, and cut-off value for IG parameters assayed by $\mathrm{XN}$ analyzer. Pairwise comparisons of the area under the ROC curves (AUCs) were performed using Z-test.

MedCalc Software 19.1 (Ostend, Belgium) and IBM SPSS Statistics 18.0.0 (Chicago, USA) were used. A value of $p<0.05$ was considered to be statistically significant.

\section{Results}

\section{Comparison of XN-derived IG\# and IG\% values and manually-derived IG\% in PB from MN, non-HN and control subjects}

Increased IG\# (both $p<0.0001$ ) and IG\% (both $p<0.0001$ ) from XN series measurements were observed in $\mathrm{MN}$ and nonHN patients when compared with healthy controls (Fig. 1). IG\% in healthy controls was not analyzed manually as the positive ratios were too low. Subgroups of patients with inflammation and receiving G-CSF showed higher levels of IG\# and IG\% from XN analysis plus IG\% from manual measurements than the entire group of non-HN patients (all $p<0.0001$; Fig. 1). Subgroups of MN patients with MPN (CML and PMF) showed the highest levels of XN-derived IG\# and IG\% values and also IG\% from manual measurements (all $p<0.0001$ ) when compared with other subgroups (Fig. 1).

\section{Correlations and differences between XN and manual estimates of IG\% in MN and non-HN patients}

Strong correlations between $\mathrm{XN}$ - and manually derived IG\% values were found in both $\mathrm{MN}$ and non-HN patients

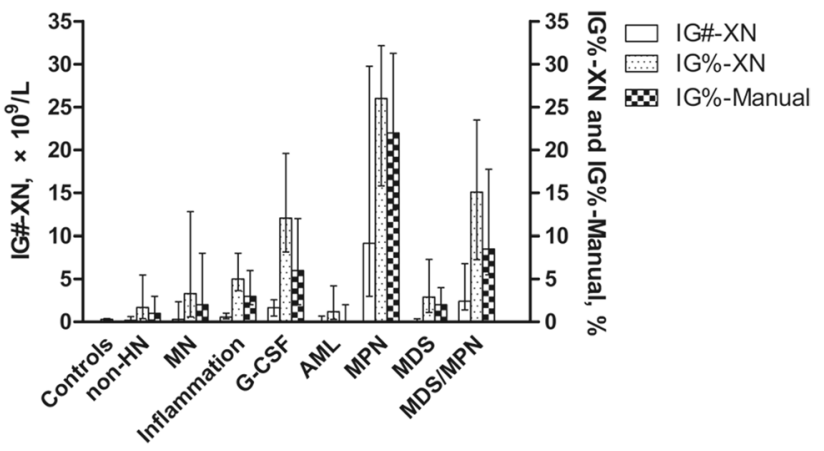

Fig. 1 Comparison of IG\#-XN, IG\%-XN and IG\%-manual values across groups. Columns and bars represent median with interquartile range

Table 2 Spearman rank correlation analysis of $\mathrm{XN}$ - and manually derived IG\% values in PB from MN and non-HN patients

\begin{tabular}{clll}
\hline & $N$ & $\begin{array}{l}\text { Correlation coef- } \\
\text { ficient }(\mathrm{r})\end{array}$ & $p$ value \\
\hline Non-HN & 524 & 0.861 & $<0.0001$ \\
$\quad$ Inflammation & 109 & 0.717 & $<0.0001$ \\
G-CSF & 68 & 0.762 & $<0.0001$ \\
MN & 388 & 0.828 & $<0.0001$ \\
AML & 231 & 0.597 & $<0.0001$ \\
MPN & 77 & 0.873 & $<0.0001$ \\
MDS & 63 & 0.767 & $<0.0001$ \\
MDS/MPN & 17 & 0.750 & 0.0005 \\
\hline
\end{tabular}

(Table 2). Although correlations were statistically significant in all groups of patients with AML, MPN, MDS, and MDS/ MPN, only moderate correlations were shown for AML patients (Table 2).

Differences between XN- and manually derived IG\% values were compared using Bland-Altman bias analysis, and differences for both $\mathrm{MN}$ and non-HN patients were considered clinically insignificant (Fig. 2). However, a relatively high difference in the mean and in the 95\% CI of limits of agreement (LoA) was shown in the G-CSF subgroup (Fig. 2).

\section{Analysis of ROC curve and cut-off values of XN IG\# and IG\% from MN and non-HN patients}

ROC analysis was performed to assess $\mathrm{XN}$-derived values of IG\# and IG\% in detection of IGs in PB. IG\% values from manual inspection were regarded as the gold standard against which the IG\# and IG\% values from $\mathrm{XN}$ measurements were compared. The AUCs of XN-derived IG\# and IG\% values were above 0.8 for both $\mathrm{MN}$ and non-HN patients, indicating a good level of detection (Table 3). 


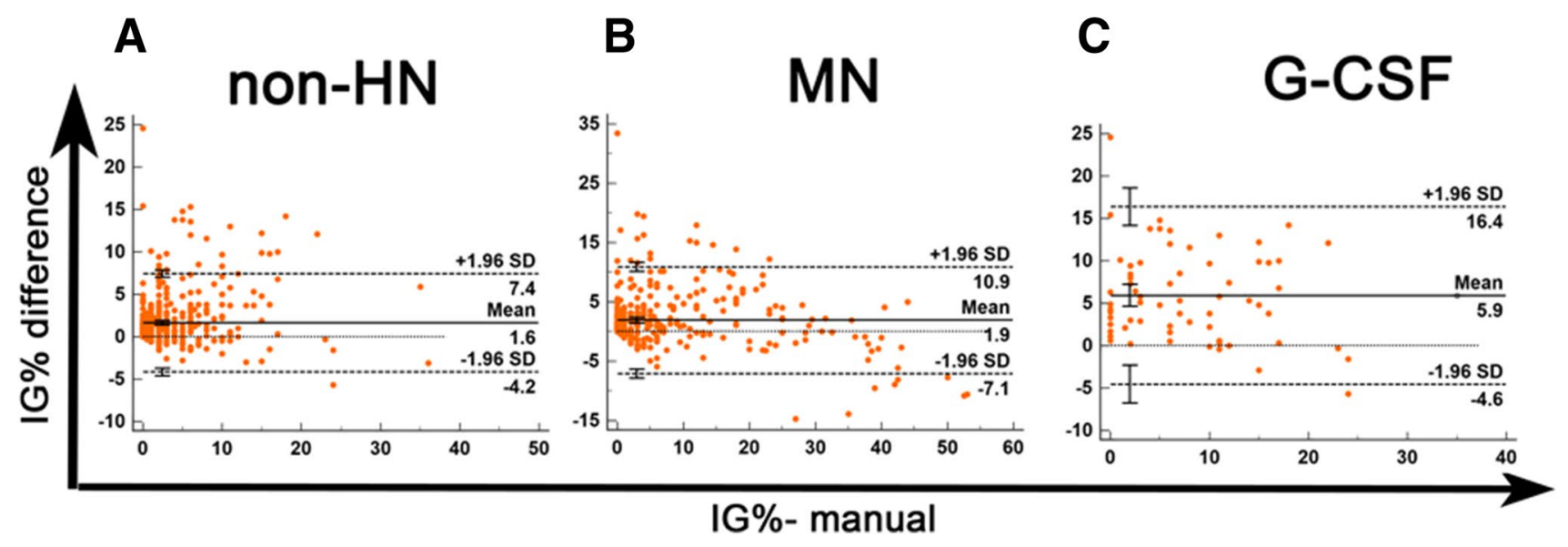

Fig. 2 Comparison of XN- and manually-derived IG\% values using Bland-Altman plot. The difference between two values (XN IG\% - manual IG\%) was plotted on the $y$-axis against manual IG\% on the $\mathrm{x}$-axis. Solid lines: mean differences; dashed lines: mean difference $\pm 1.96 \mathrm{SD}$

Comparison of the AUCs for XN-derived IG\# and IG\% values for MN patients revealed that the $\mathrm{IG} \%$ values showed better efficiency than IG\# $(p<0.05)$. Both the XN-derived IG\# and IG\% parameters showed higher efficiency in non$\mathrm{HN}$ than in MN patients $(p<0.05)$. The Youden index gave cut-off values of $0.200 \times 10^{9} / \mathrm{L}$ for IG\# and $1.95 \%$ for IG\% (Table 3).

\section{Discussion}

IGs are often found in the PB of patients with acute infection, inflammation, acute hemorrhage, and those receiving G-CSF. The non-HN patients enrolled in the current study included a number of patients with these conditions accounting for the finding that IG levels among non-HN patients were higher than those of controls.

IGs are easily distinguishable from mature leukocytes in the WDF channel due to their larger nuclear volume and higher fluorescent intensity. This observation underlies the strong correlations between IG\% values measured by $\mathrm{XN}$ and those from manual inspection in non-HN and $\mathrm{MN}$ PB samples. However, morphological abnormalities of immature and mature granulocytes confuse the identification of IGs, resulting in inaccurate counts.

Overall comparisons of WDF scattergrams revealed that inconsistencies between IG\% values from XN and manual measurements were usually due to inaccurate IG gating in the scattergram (Supplemental Fig. S1). Granulocytes with morphological abnormalities could be identified in blood smears from all of the inconsistent cases (Supplemental Fig. S2). This phenomenon was observed among samples from patients with acute promyelocytic leukemia (APL), acute myelomonocytic leukemia (AML-M4), and acute monoblastic and monocytic leukemia (AML-M5). The 2015 International Council for Standardization in Haematology (ICSH) recommended that abnormal promyelocytes in APL samples should be counted as blast equivalents rather than as IGs [1]. In addition, neutrophil dysplasia, such as hypogranulation and hyposegmentation, may also be present in AML samples $[1,11]$ and might interfere with IG gating, leading to inconsistencies. Moreover, "left shift", arising from elevation of percentages of band neutrophils $(>5 \%)$ and cells with hypergranulation, vacuolation, and cytosolic Döhle bodies [1, 21], often follows G-CSF administration and may interfere with the intelligent software system gating and falsely raise IG count.

Table 3 ROC curve and cut-off values for XN-derived IG\# and IG\% values in PB from MN and non-HN patients

\begin{tabular}{llllllllll}
\hline & $\mathrm{N}$ & AUC & SE & $95 \%$ CI $^{\mathrm{a}}$ & $p$ value & Cut-off & Sensitivity & Specificity & Youden index \\
\hline IG\#-XN, $\times 10^{9} / \mathrm{L}$ & & & & & & 0.200 & & & \\
$\quad$ Non-HN & 524 & 0.918 & 0.013 & 0.892 to 0.943 & $<0.0001$ & & 0.767 & 0.886 & 0.653 \\
$\quad$ MN & 388 & 0.842 & 0.002 & 0.803 to 0.881 & $<0.0001$ & & 0.746 & 0.791 & 0.537 \\
IG\%-XN, \% & & & & & & 1.95 & & & \\
Non-HN & 524 & 0.937 & 0.011 & 0.916 to 0.958 & $<0.0001$ & & 0.787 & 0.934 & 0.721 \\
MN & 388 & 0.885 & 0.017 & 0.851 to 0.918 & $<0.0001$ & & 0.813 & 0.784 & 0.597 \\
\hline
\end{tabular}


In summary, the IG parameters derived from Sysmex $\mathrm{XN}$ hematology analyzer efficiently indicate the presence of IG in PB of MN patients, and this approach may facilitate screening for MNs. In addition, inaccuracies in $\mathrm{XN}$-derived IG parameter measurements may arise due to the presence of granulocytes with morphological abnormalities in $\mathrm{PB}$ samples.

Supplementary Information The online version contains supplementary material available at https://doi.org/10.1007/s12308-022-00484-w.

Acknowledgements The authors would like to express their gratitude to EditSprings (https://www.editsprings.cn/) for the expert linguistic services provided.

Author contribution Yajuan Shen conceptualized, designed, and supervised the study and also drafted the manuscript; Qifeng Lu analyzed data and edited the manuscript; Tian Li and Tingting Hou provided interpreting data; Ying Li participated in discussing the conception and manuscript; Yajuan Zhao, Shu Feng, Xixian Yang, and Mengyu Zhu sorted out data.

Funding This work was supported by Natural Science Foundation of Shandong Province, China, under Grant (No.ZR2017MH088).

Availability of data and material Available on request.

\section{Declarations}

Ethics approval This study was approved by the medical Ethics Committee of Shandong Provincial Hospital (No.2016-KY-051).

Consent to participate This study was conducted only after taking informed consent from all participants.

Consent for publication All the authors gave their consent for submission/publication of this manuscript.

Conflict of interest The authors declare no competing interests. 95\% $\mathrm{CI}^{a} 95 \%$ confidence intervals.

Open Access This article is licensed under a Creative Commons Attribution 4.0 International License, which permits use, sharing, adaptation, distribution and reproduction in any medium or format, as long as you give appropriate credit to the original author(s) and the source, provide a link to the Creative Commons licence, and indicate if changes were made. The images or other third party material in this article are included in the article's Creative Commons licence, unless indicated otherwise in a credit line to the material. If material is not included in the article's Creative Commons licence and your intended use is not permitted by statutory regulation or exceeds the permitted use, you will need to obtain permission directly from the copyright holder. To view a copy of this licence, visit http://creativecommons.org/licenses/by/4.0/.

\section{References}

1. Palmer L, Briggs C, McFadden S, Zini G, Burthem J, Rozenberg $\mathrm{G}$ et al (2015) ICSH recommendations for the standardization of nomenclature and grading of peripheral blood cell morphological features. Int J Lab Hematol 37:287-303

2. Fowlie PWSB (1998) Diagnostic tests for bacterial infection from birth to 90 days-a systematic review. Arch Dis Child Fetal Neonatal Ed 78:F92-98

3. Fernández-Suárez APV, Gimenez MT, Hernández JF (2003) Immature granulocyte detection by the SE-9000 haematology analyser during pregnancy. Clin Lab Haematol 25:347-351

4. Blazkova J, Gupta S, Liu Y, Gaudilliere B, Ganio EA, Bolen CR et al (2017) Multicenter systems analysis of human blood reveals immature neutrophils in males and during pregnancy. J Immunol 198:2479-2488

5. Nigro KG, O'Riordan M, Molloy EJ, Walsh MC, Sandhaus LM (2005) Performance of an automated immature granulocyte count as a predictor of neonatal sepsis. Am J Clin Pathol 123:618-624

6. Cimenti C, Erwa W, Herkner KR, Kasper DC, Muller W, Resch B (2012) The predictive value of immature granulocyte count and immature myeloid information in the diagnosis of neonatal sepsis. Clin Chem Lab Med 50:1429-1432

7. Karon BS, Tolan NV, Wockenfus AM, Block DR, Baumann NA, Bryant SC et al (2017) Evaluation of lactate, white blood cell count, neutrophil count, procalcitonin and immature granulocyte count as biomarkers for sepsis in emergency department patients. Clin Biochem 50:956-958

8. Yoo J, Ahn SS, Jung SM, Song JJ, Park YB, Lee SW (2018) Delta neutrophil index is associated with vasculitis activity and risk of relapse in ANCA-associated vasculitis. Yonsei Med J 59:397-405

9. Zhong H, Yazdanbakhsh K (2018) Hemolysis and immune regulation. Curr Opin Hematol 25:177-182

10. Park SH, Park CJ, Kim MJ, Han MY, Lee BR, Cho YU et al (2016) Evaluation of parameters obtained from the Sysmex $\mathrm{XN}-2000$ for predicting the recovery of the absolute neutrophil count and platelets after hematopoietic stem cell transplantation. Int J Lab Hematol 38:198-208

11. Arber DA, Orazi A, Hasserjian R, Thiele J, Borowitz MJ, Le Beau MM et al (2016) The 2016 revision to the World Health Organization classification of myeloid neoplasms and acute leukemia. Blood 127:2391-2405

12. Carulli G (1997) Effects of recombinant human granulocyte colony-stimulating factor administration on neutrophil phenotype and functions. Haematologica 82:606-616

13. Vadhan-Raj SKM, LeMaistre A, Hittelman WN, McCredie K, Trujillo JM, Broxmeyer HE et al (1987) Effects of recombinant human granulocyte-macrophage colony-stimulating factor in patients with myelodysplastic syndromes. N Engl J Med 317:1545-1552

14. Field D, Taube E, Heumann S (2006) Performance evaluation of the immature granulocyte parameter on the Sysmex XE-2100 automated hematology analyzer. Lab Hematol 12:11-14

15. Seo JY, Lee ST, Kim SH (2015) Performance evaluation of the new hematology analyzer Sysmex XN-series. Int J Lab Hematol 37:155-164

16. Redin ME, Vives A, Garrido A, Urrechaga E, Lacasta M (2016) Local verification between the hematological analyzers Sysmex XN-series and XE-5000. Int J Lab Hematol 38:256-264

17. Schoorl M, Schoorl M, Chevallier M, Urrechaga E, Lacasta M (2017) Multicenter verification of the Sysmex XN-Series. Int J Lab Hematol 39:489-496

18. Swerdlow SH, Campo E, Pileri SA, Stein H, Siebert R, Advani R et al (2016) The 2016 revision of the World Health Organization classification of lymphoid neoplasms. Blood 127:2375-2390

19. Clinical Laboratory Standards Institute (2007) Reference leukocyte (WBC) differential count (proportional) and evaluation of instrumental methods; approved standard, 2nd edn, Wayne, CLSI 
20. Mukaka MM (2012) Statistics corner: A guide to appropriate use of correlation coefficient in medical research. Malawi Med J 24:69-71

21. Prokocimer M, Potasman I (2008) The added value of peripheral blood cell morphology in the diagnosis and management of infectious diseases-part 1: basic concepts. Postgrad Med J 84:579-585

22. Maenhout TM, Marcelis L (2014) Immature granulocyte count in peripheral blood by the Sysmex haematology XN series compared to microscopic differentiation. J Clin Pathol 67:648-650

23. Briggs CJ, Linssen J, Longair I, Machin SJ (2011) Improved flagging rates on the Sysmex XE-5000 compared with the XE-2100 reduce the number of manual film reviews and increase laboratory productivity. Am J Clin Pathol 136:309-316

Publisher's note Springer Nature remains neutral with regard to jurisdictional claims in published maps and institutional affiliations. 